\title{
Review: adderall may have a small advantage over methylphenidate in attention deficit hyperactivity disorder
}

\author{
Faraone SV,Biederman J, Roe C. Comparative efficacy of adderall and methylphenidate in attention deficit hyperactivity \\ disorder: a meta-analysis. J Clin Psychopharm 2002 Oct;22:468-73.
}

QUESTION: How effective is adderall compared with methylphenidate in attention deficit hyperactivity (ADHD) disorder?

\section{Design}

Review with meta-analysis.

\section{Data sources}

Studies were identified using Pubmed. The authors do not provide details of any additional search strategies.

\section{Study selection}

Trials comparing adderall with methylphenidate for children with ADHD (DSM-IV criteria) were eligible if (1) outcome assessors were blind to treatment allocation; (2) means and standard deviations were presented for outcome scores; (3) structured methods were used to assess ADHD symptoms, aggressive or defiant symptoms or global ratings of behaviour. The authors included 2 clinic-based studies and 2 based at summer camps. Participants were 216 children aged 5-17 years.

\section{Data extraction}

Data were extracted on symptoms and behaviour scores reported by clinicians, parents and teachers. Standardised mean differences were calculated for each outcome. No data suitable for meta-analysis were available on duration of treatment effect.

\section{Main results}

Adderall had a small advantage over standard-release methylphenidate on both symptom measures and global ratings (table). Clinician and parent ratings of adderall were superior to methylphenidate. There was no significant difference in teacher ratings.

Pooled analysis of adderall and methylphenidate in ADHD

\begin{tabular}{lll} 
& & \multicolumn{1}{c}{$\begin{array}{c}\text { Pooled } \\
\text { standardised } \\
\text { mean difference } \\
\text { between groups }\end{array}$} \\
Symptom type & ADHD symptoms & 0.17 \\
\hline & $\begin{array}{l}\text { Aggressive / } \\
\text { defiant symptoms }\end{array}$ & $0.23^{*}$ \\
\hline & $\begin{array}{l}\text { Global rating } \\
\text { symptoms }\end{array}$ & $0.52^{*}$ \\
\hline Type of rater & Clinician & $0.37^{*}$ \\
\hline & Parent & $0.28^{*}$ \\
\hline Type of dosing & Teacher & 0.13 \\
\hline & Best dose & $0.32^{*}$ \\
\hline & Fixed dose & $0.22^{*}$
\end{tabular}

Note: Positive mean differences indicate that adderall was superior to methylphenidate. Statistically significant differences $(p \leq 0.05)$ are marked *

\section{Conclusions}

Adderall is at least as effective as standard-release methylphenidate for attention deficit hyperactivity disorder and may be marginally better.

\section{COMMENTARY}

Faraone et al accounted for confounding in the controlled comparison studies between amphetamine and methylphenidate, including the use of different outcome measures, dosing regimens and concomitant exposure to intensive behavioural and psychosocial treatments. This type of analysis is necessary given the paucity of adequately powered, controlled comparison studies between amphetamine-based and methylphenidate-based stimulants. It is also unlikely that commercial funding for such studies will be readily available in the future, given the interest in developing non-stimulant compounds for ADHD.

The questions raised by this analysis are of vital importance to clinicians. Given the proliferation of new stimulant treatments for $\mathrm{ADHD}$, is there a scientific basis for choosing one subtype of stimulant over another in newly diagnosed ADHD? Even a small difference in efficacy between compounds may have a significant clinical impact given the number of people treated for $\mathrm{ADHD}$ and the long-term functional impairment associated with the disorder. This analysis also calls into question the "gold standard" for ADHD pharmacotherapy. Should adderall be the standard to which new ADHD treatments are compared (rather than methylphenidate)? The analysis also raises awareness that selecting a specific psychostimulant has significant implications for outcomes in individual patients. A previous meta-analysis by Arnold $e t$ al found that a small majority of participants responding to stimulants had a preferential response to one subtype of stimulant over another, with a higher percentage exhibiting preferential response to amphetamine. ${ }^{1}$

When viewed in the context of previous literature, this analysis suggests that adderall has small but significant advantages over methylphenidate. Faraone $e t$ al suggest that future studies may be more useful if both symptom ratings and global ratings are used as outcome measures. Symptom ratings help to assess whether a compound has clinically significant effects, but another, often ignored, factor involves the robustness of the response to treatment. Is one compound more likely than another to lead to remission of symptoms? Functional improvement needs to be evaluated in clinical trials along with symptom reduction. Stephen Grcevich, MD Division of Child and Adolescent Psychiatry Case Western Reserve University School of Medicine Cleveland, Ohio, USA

1 Arnold LE. Methylphenidate vs. amphetamine: A comparative review. J Attent Disord 2000; 3: 200-11.
Source of funding. Shire Richwood Pharmaceuticals.

For correspondence: SV Faraone, Pediatric Psychopharmacology Unit, Boston, USA. sfaraone@hms.harvard.edu 